\title{
Contingency severity assessment for voltage security using non-parametric regression techniques
}

\author{
L. Wehenkel \\ Senior Research Assistant F.N.R.S. - Dept. of Electrical Engineering - Institut Montefiore \\ University of Liège - Sart Tilman B 28, B 4000 Liège - Belgium
}

\begin{abstract}
This paper proposes a novel approach to voltage security assessment exploiting non-parametric regression techniques to extract simple and at the same time reliable models of the severity of a contingency, defined as the difference between pre- and postcontingency load power margins. The regression techniques extract information from large sets of possible operating conditions of a power system screened off-line via massive random sampling, whose voltage security with respect to contingencies is pre-analyzed using an efficient voltage stability simulation. In particular, regression trees are used to identify the most salient parameters of the pre-contingency topology and electrical state which influence the severity of a given contingency, and to provide a first guess transparent approximation of the contingency severity in terms of these latter parameters. Multilayer perceptrons are exploited to further refine this information. The approach is demonstrated on a realistic model of a large scale voltage stability limited system, where it shows to provide valuable physical insight and reliable contingency evaluation. Various potential uses in power system planning and operation are discussed.
\end{abstract}

Keywords - Voltage security; load power margin; computer based learning; regression trees; artificial neural networks.

\section{INTRODUCTION}

Many recent large-scale power system breakdowns have been the consequence of instabilities characterized by sudden voltage collapse phenomena.

One of the main reasons for this is that transient stability limits of power flows have increased, consequently to the improvements of protections as well as static var compensators and generator speed and voltage regulators. Thereby more power may be transferred over longer distances and systems which used to be transient stability limited become voltage stability limited. This situation has been observed in North-American and European power systems and has been a major incentive to research on voltage stability. Although recent advances are impressive there are still open questions, in particular as concerning the definition of widely accepted models and security criteria. We refer the reader to $[1,2,3]$ for an overview of the concepts and industry experience in this field.

Voltage security concerns mainly the ability of a system to control its EHV voltage while submitted to various contingencies, in particular to outages and rapid load build up. In power system operation a useful concept is the load power margin (LPM); this is a security margin expressed in terms of the

PAPER NO 95WM162-8 PWRS ACCEPTED FOR PRESENTATION AT THE 1995 IEEE PES WINTER MEETING amount of additional load (P and $\mathrm{Q}$ ) which may be supplied by the system under acceptable conditions. Various "exact" or "approximate" approaches have been proposed in the literature to compute this kind of margin $[4,5,6,7]$, with computing times of the order of several power flow computations. The direct physical interpretation of the LPM makes it an easily accepted tool by operators. Note also that in practice there is often a good correlation among LPMs obtained by different ways, which suggests that they may be interpreted as a distance to insecurity.

For a given power system, the available LPM depends on its topology, load level, and available reactive generation and compensation resources, while the amount of margin required depends on the actual load trend. Thus, the system will be considered as secure if it is able to withstand all credible contingencies with sufficient post-contingency LPM. While many factors may influence the pre- and post-contingency LPM, we conjecture that for a given contingency in most circumstances only a rather small number of system parameters will actually influence its severity, i.e. the difference of its pre- and post-contingency LPMs. In other words, voltage security assessment should be decomposed into (i) the computation of the pre-contingency LPM once per operating state, using any of the above computational techniques, and (ii) the evaluation of contingency severities, where simplified approximate models may be used. We shall discuss and illustrate this conjecture and propose a systematic approach to derive a simple but reliable assessment of the contingency severity.

In particular, to obtain a prediction of the impact of a contingency on the LPM, without requiring on-line the recomputation of the post-contingency margin, we propose to use statistical regression techniques. These techniques exploit large random samples of operating conditions simulated off-line (to precompute pre- and post-contingency LPMs) in order to identify the main factors which influence the severity of a contingency and to build approximate simplified models of its post-contingency LPM. These approximate models are easy to exploit on-line and may thus be used for contingency ranking and evaluation, and for determining dangerous combinations of contingencies and even control actions for security enhancement.

The remainder of the paper is organized as follows. In section 2 we introduce the overall regression based framework to voltage security assessment, and briefly describe the complementary regression tree and multilayer perceptron techniques. Section 3 provides an overview of a case study carried out on the Brittany region of the EHV system of Electricite de France, so as to assess the approach in the context of three typical contingencies. Before concluding, we discuss in section 4 the various possible uses of the approach for off-line studies and on-line operation.

\section{LOAD POWER MARGIN REGRESSION}

Figure 1 highlights the overall voltage security framework.

For a given power system and voltage security problem, operating states are first generated via random sampling, in a suf- 


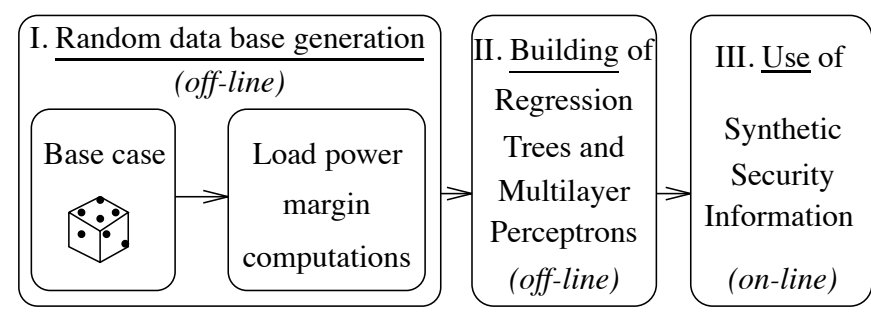

Figure 1. Voltage security framework

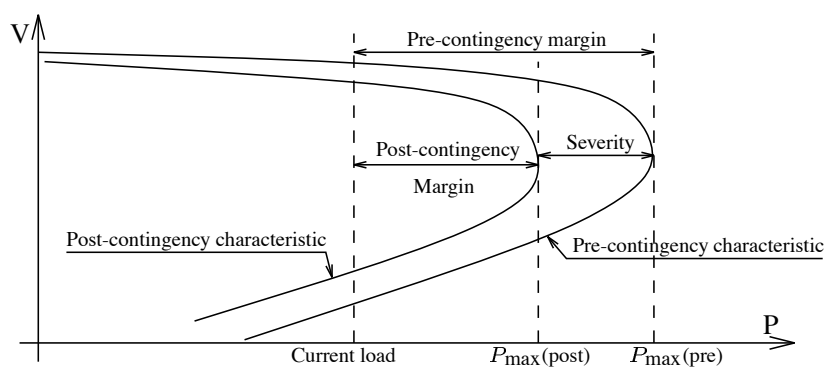

Figure 2. Typical EHV PV transmission characteristics

ficiently broad and diverse range so as to screen all situations deemed relevant. Each case is characterized by pre-contingency attributes, i.e. parameters describing its electrical and topological normal state. Further, it is pre-analyzed in terms of voltage security by computing its pre- and post-contingency LPMs, for various contingencies. Typically, several thousand operating states are drawn and several tens of contingencies are considered in a given study. Massive parallelism may be exploited to speed up this otherwise lengthy off-line simulation phase.

At the second step, the non-parametric regression techniques are applied, in a contingency by contingency fashion, to extract synthetic security information in the form of approximate models expressing the relationship between the attributes and the severity of a contingency, which is defined by the drop in LPM from pre-contingency to the post-contingency state (see Fig. 2). In this context, regression trees are used to identify salient attributes which influence the severity of a contingency and to provide a first guess transparent model, while multilayer perceptrons are used to refine this model.

The third step consists of using this synthetic security information on-line for security assessment.

\subsection{Contingency severity for voltage security}

We denote by $\left(T_{0}, X_{0}\right)$ the pre-contingency topology and electrical state and $\mathrm{Ma}_{0}\left(T_{0}, X_{0}\right)$ the pre-contingency margin.

Further, we denote by $\mathrm{Ma}_{i}\left(T_{0}, X_{0}\right)$ the post-contingency load power margin when contingency $i$ is applied to the state $\left(T_{0}, X_{0}\right)$, and we thus define by

$$
\Delta \operatorname{Ma}_{i}\left(T_{0}, X_{0}\right) \triangleq \operatorname{Ma}_{0}\left(T_{0}, X_{0}\right)-\operatorname{Ma}_{i}\left(T_{0}, X_{0}\right)
$$

the severity (in state $\left(T_{0}, X_{0}\right)$ ) of the contingency.

In the same fashion, denoting by $\left(T_{0, i_{1}, \ldots, i_{k-1}}, X_{0, i_{1}, \ldots, i_{k-1}}\right)$ the topology and electrical state of a system following $k-1$ outages, the severity of a sequence of $k$ outages is derived by

$$
\begin{aligned}
\Delta \mathrm{Ma}_{i_{1}, \ldots, i_{k}}\left(T_{0},\right. & \left.X_{0}\right)=\Delta \mathrm{Ma}_{i_{1}, \ldots, i_{k-1}}\left(T_{0}, X_{0}\right) \\
& \left.+\Delta \mathrm{Ma}_{i_{k}}\left(T_{0, i_{1}, \ldots, i_{k-1}}, X_{0, i_{1}, \ldots, i_{k-1}}\right)\right) .
\end{aligned}
$$

The latter formula expresses the fact that if we are able to predict the security margin for a single outage in terms of parameters whose changes in the post-contingency state are easy to evaluate

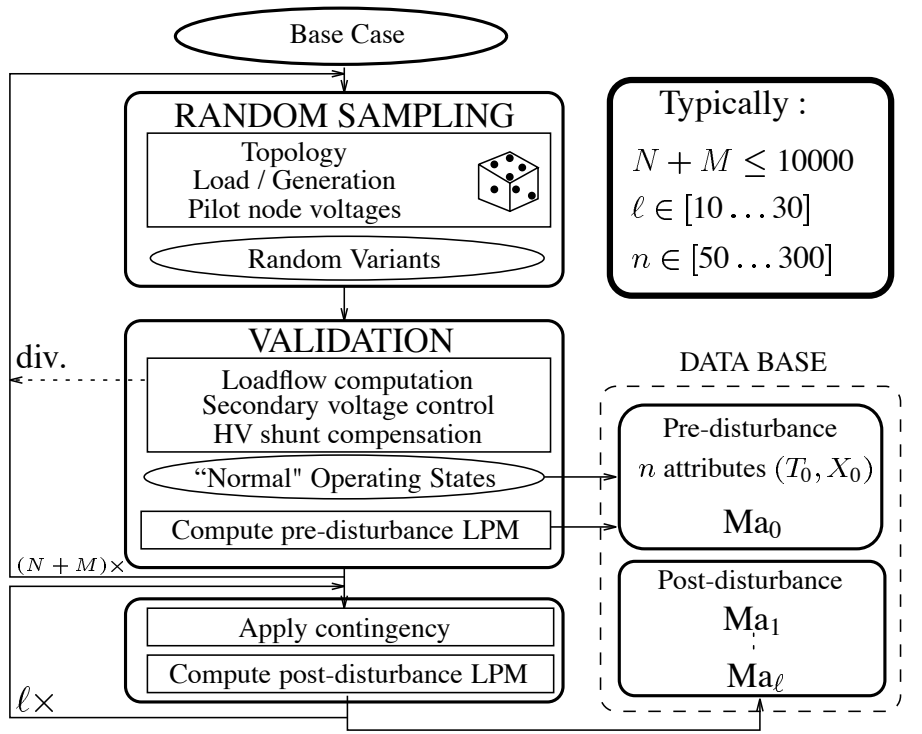

Figure 3. Principle of the data base generation

then we are also able to predict the effect of any sequence of outages.

To construct automatically an approximation of the term $\Delta \mathrm{Ma}_{i}(T, X)$, we propose to use computer based learning techniques in the form of non-parametric statistical regression methods, using representative learning sets of randomly generated power system operating states, pre-characterized with respect to voltage security by their pre- and post-disturbance LPM.

\subsection{Random generation of a data base}

Computer based learning techniques need a representative sample of power system situations, for which topology $T_{0}$, electrical state $X_{0}$ and margins $\mathrm{Ma}_{0}$ and $\mathrm{Ma}_{i}$ are pre-determined. The construction of such samples calls for random sampling techniques similar to those developed in the decision tree approach to power system security assessment [8], and exploits an appropriate "system-theory" LPM computation method.

The overall principle of such a data base generation is depicted in Fig. 3, where we consider a total number of $\ell$ different contingencies, $n$ different candidate attributes, and an overall sample of $N+M$ operating states, where $N$ denotes the number of states used in the learning set to derive the approximate models, and $M$ the number of independent test states used subsequently to validate them.

The random sampling approach aims at screening all relevant power system situations, and in particular normal (usual) states as well as weak situations. The power system engineers are generally able to provide valuable prior information helping to determine the considered random variations (e.g. load level, generation schedule, topology, voltage set points ...).

\subsection{Formulation of the regression problem}

In the above equation (1) $\mathrm{Ma}_{0}$ is the contingency independent term while the two other terms are in practice strongly contingency dependent, and it is clearly appropriate to build the regression models in a single-contingency approach so as to exploit the latter specificity.

Thus, for a given contingency we select its learning set $(L S)$ as the relevant operating states (denoted $o$ below) for this contingency among the $N$ first of the data base. Each state is characterized by : (i) $n$ candidate attributes $a_{1}(o), \ldots, a_{n}(o)$ describing its topology (e.g. in/out indicators) and electrical state (e.g. voltages, power flows, generation levels, reactive 


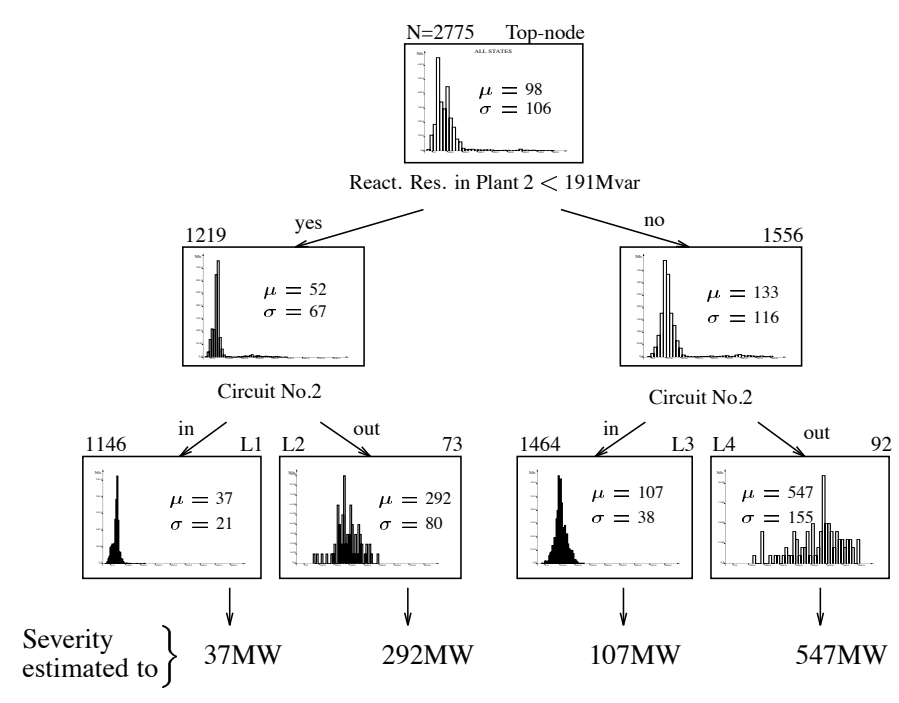

Figure 4. Regression tree for line tripping contingency

reserves ... ) which are deemed to influence the severity of the contingency and in terms of which it is desired to express the regression models; (ii) its difference $\Delta \mathrm{Ma}_{i}(o)$ of its pre-computed values of $\mathrm{Ma}_{0}(o)$ and $\mathrm{Ma}_{i}(o)$.

Then the learning objective is to build an approximate model,

$$
\Delta \tilde{\mathrm{Ma}}{ }_{i}(o)=F_{i}\left(a_{1}(o), \ldots, a_{n}(o)\right),
$$

where the function $F_{i}$ is determined so as to "explain" as much as possible the variance of $\Delta \mathrm{Ma}_{i}$ observed in the learning set, e.g. such that the Mean Square Error (MSE)

$$
M S E\left(F_{i}\right) \triangleq \sum_{o \in L S}\left\|\Delta \mathrm{Ma}_{i}(o)-F_{i}\left(a_{1}(o), \ldots, a_{n}(o)\right)\right\|^{2},
$$

is as small as possible. Notice that this calls for the identification among the candidate attributes of a subset of attributes which are actually relevant, i.e. which actually influence the severity of the particular contingency under consideration. We conjecture that for each contingency it is possible to identify a small number of attributes able to explain most of the variance of its severity. Obviously, these salient attributes are liable to change significantly from one contingency to another.

\subsection{Non-parametric regression techniques}

It would be hopeless to use a simple parametric (e.g. linear or quadratic) family of regression functions $F_{i}$ in order to treat the wide variety of voltage security problems, which are highly nonlinear. Thus we turn to non-parametric regression techniques able to cope with a broad range of non-linearities. According to our experience, regression trees and multilayer perceptrons are two efficient and complementary such approaches [9]. Below we describe the former and merely summarize the advantages and drawbacks of the latter better known approach.

\subsubsection{REGRESSION TREES [10]}

Similarly to decision (or classification) trees, regression trees decompose the attribute space into a hierarchy of regions. In our application they will decompose the pre-disturbance operating space of a power system into regions where the severity of a contingency is as constant as possible. In each such region the severity will be estimated by its expected value determined in the corresponding learning sub-sample.

Similarly to decision trees, regression trees are built in a topdown approach : starting with the top-node (e.g. see Fig. 4) and the complete learning set, an attribute $a_{i}$ and a threshold value $v_{i}$ are selected to decompose the learning set into two subsets, corresponding to states for which $a_{i}<v_{i}$ and $a_{i} \geq v_{i}$ respectively. The split is determined so as to reduce as much as possible the severity variance in the subsets, or in other words to provide a maximum amount of information on the severity.

The procedure continues splitting until either the variance has been sufficiently reduced or it is not possible to reduce it further in a statistically significant way. The latter may happen due to either a reduced subsample size or a low predictive value of the candidate attributes at a tree node.

In our simulations we have used the method described in [8] together with its post-pruning algorithm to avoid overfitting.

For prediction, the regression tree is used similarly to a decision tree : a new state is directed through the tree, by starting at the top-node and applying the encountered tests to direct the state towards the appropriate successor. When a terminal node is reached, the there stored mean value (or confidence interval) is used as an estimate of the severity. It thus provides a piecewise constant model which is particularly well adapted to represent the effect of topology as well as electrical state. On the other hand, due to its interpretability and capability to identify the attributes which influence most strongly the severity, it is an appropriate data analysis tool for validation.

The main practical difference between decision and regression trees is that the latter determine automatically the appropriate quantization of the severity into subintervals, whereas the former merely reproduce a predefined classification.

\subsubsection{MULTILAYER PERCEPTRONS [11, 12]}

Multilayer perceptrons provide an easy to use and flexible technique for non-linear regression. They are defined by their topology, in terms of layers of neurons, and the values of weights and thresholds. A potential difficulty may result from their black box nature and corresponding lack of interpretability, especially if the number of input parameters is large. For this reason, as well as for computational efficiency, we deem it appropriate to preselect a reduced number of input attributes for the multilayer perceptron by first building a regression tree for the considered contingency. On the other hand, multilayer perceptrons show potential in reducing the approximation error of regression trees, due to their continuous modelling capabilities.

Due to space limitations, we refer the interested reader to $[11,12]$ for further information on this by now popular technique. We merely mention that in our experiments we have used single hidden layer perceptrons with sigmoidal activation functions and a batch quasi-Newton "Broyden-Fletcher-GoldfarbShanno" (BFGS) iterative optimization algorithm to minimize the MSE of eqn. (4).

\section{CASE STUDY}

Below we summarize a broad study carried out on the EHV system of Electricité de France (EDF), commenting briefly on the physical problem and the overall scope of the study, in terms of operating conditions and contingencies, before discussing the case of three contingencies of increasing complexity.

\subsection{A real-life voltage security problem [13]}

Figure 5 depicts the one-line diagram of part of the EDF system, covering the study region of Brittany and the surrounding relevant part. A broad voltage security study is in progress on the basis of this model, for which a data base composed only of 5000 normal operating states was generated. 


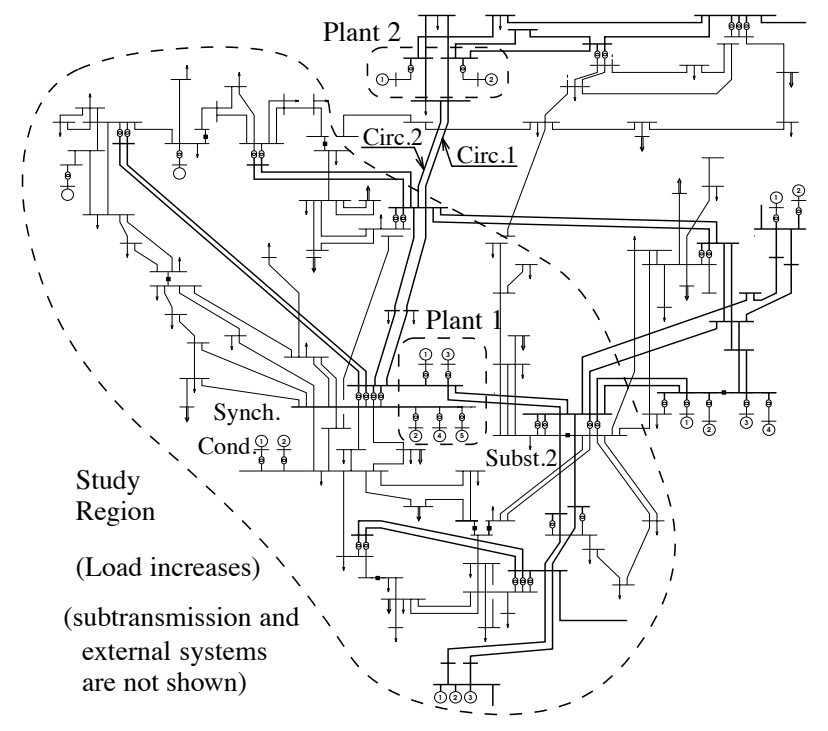

Figure 5. Brittany region one-line diagram $(225 \mathrm{kV}+400 \mathrm{kV})$

\subsubsection{OPERATING CONDITIONS AND CONTINGENCIES}

To save space, we merely quote the independent variables used during the random sampling of the pre-disturbance states : topology ( single or double line $(400 \mathrm{kV}$ or $225 \mathrm{kV})$ or transformer outages); load level (including randomization of individual HV load distribution, power factors, compensation and voltage sensitivities); regional unit commitment (with variable active power generation levels in Plant 1); reactive support (synchronous condenser; EHV and HV shunt compensation; gas turbines) and secondary voltage control set-points.

A total of 13,513 random variants were drawn to yield 5000 pre-disturbance states. (The remaining 8,513 variants led to power flow computation divergence or non-convergence.) For each state about 200 attributes were computed, corresponding to key variables such as topological indicators, important EHV power flows, $400 \mathrm{kV}$ voltages, numbers of units in operation in power plants, total load demand, reactive shunt compensation reserves in the study region, and reactive generation reserves.

All in all 26 different contingencies were considered in this broad study, corresponding to synchronous condenser, generator or line tripping and busbar faults. Thus, in addition to the pre-contingency LPM, the corresponding 26 post-contingency LPMs were computed for each operating state, yielding a total number of 135,000 LPM computations!

\subsubsection{ELECTRICAL MODELLING AND MARGINS}

The electrical static and dynamic models, the voltage security criterion and the LPM computation procedure used are discussed in detail in [7]. We merely outline their salient characteristics to indicate their highly realistic character.

A 1200-bus modelling including subtransmission levels and distribution feeders is used while secondary voltage control is modelled both in the static and dynamic computations. As concerns the latter, a simplified mid-term voltage stability simulation is carried out to track the on-load-tap-changers, automatic shunt compensation, machine overexcitation limiters and coordinated secondary voltage control behavior, subsequently to a disturbance.

Pre- and post-disturbance LPMs are computed by simulating the system response if submitted to a steady load increase until a voltage instability is reached. The latter is identified according to the change in sign of the sensitivity of total reactive power generation to individual $\mathrm{HV}$ reactive demands.

The standard deviation of the numerical computation error
Table 1. LPM computation error vs classification error

\begin{tabular}{|c|c|c|}
\hline Noise & \multicolumn{2}{|c|}{ Classification error rate } \\
\cline { 2 - 3 } standard deviation & Gaussian distribution & Uniform distribution \\
\hline \hline $10 \mathrm{MW}$ & $1.23 \%$ & $1.45 \%$ \\
$15 \mathrm{MW}$ & $1.71 \%$ & $1.88 \%$ \\
$20 \mathrm{MW}$ & $2.17 \%$ & $2.28 \%$ \\
$30 \mathrm{MW}$ & $3.33 \%$ & $3.40 \%$ \\
$100 \mathrm{MW}$ & $10.67 \%$ & $11.54 \%$ \\
\hline
\end{tabular}

of the margin was estimated to about $15 \mathrm{MW}$. Further, for a fixed regional load level, the standard deviation of the margin variation due to uncertainty in the load distribution as modelled in the data base is larger than 60MW. This estimates the LPM accuracy obtained via "exact" numerical computations. Using the latter to classify operating states of the data base, this uncertainty translates into classification errors in a way depending on the density of states in the neighborhood of the classification threshold. E.g., Table 1 shows the relationship between LPM computation noise and error rates, for a contingency corresponding to the loss of a generator in Plant 1 , and classification with respect to a LPM threshold of $255 \mathrm{MW}$. The error rates are obtained by comparing the classification obtained by the computed margin, with the classification obtained by adding a noise term (assuming either a Gaussian or a uniform random distribution) to the computed margin. The figures are mean values and their standard deviation resulting from 20 passes through the data base with different random seeds.

\subsection{Contingency severities}

Table 2 summarizes the statistical information concerning the post-contingency margins of 3 contingencies discussed below, as well as their severity measured by $\Delta \mathrm{Ma}_{i}=\mathrm{Ma}_{0}-\mathrm{Ma}_{i}$. The first column describes the contingency, the second column shows the number $N+M$ of relevant states for this contingency among the 5000, while the third and fourth (resp. fifth and sixth) columns indicate the mean and standard deviation of the postcontingency margin (resp. severity) in the data base.

As a ground for comparison, the first line of Table 2 shows the mean and standard deviation of the pre-contingency margins computed for the 5000 states of the data base. We mention that the overall load level in the region shown in Fig. 5 varies between $5000 \mathrm{MW}$ and $7700 \mathrm{MW}$, while the pre-contingency LPM in this region varies between 0 and $1750 \mathrm{MW}$, with a mean of $756 \mathrm{MW}$ and a rather high standard deviation of $325 \mathrm{MW}$, indicating the diversity of situations covered in the data base.

The scatter plots shown in Fig. 6, show the distribution of pre- vs post-contingency LPM in the data base. Each point represents an operating state, and its vertical distance from the diagonal is equal to the severity of the contingency for this state. Thus the farther the location of the cloud center below the diagonal the higher the mean severity of the contingency and the higher the spread of the points the more variable the severity from case to case.

\subsection{Severity assessment}

\subsubsection{SYNCHRONOUS CONDENSER TRIPPING}

Clearly, the tripping of the (300MVA) synchronous condenser is a rather mild and quite simple contingency (see Fig. 6a). Its

Table 2. Statistics of post-contingency margins and severities

\begin{tabular}{|c|c||cc|rr|}
\hline $\begin{array}{c}\text { Description } \\
\text { of }\end{array}$ & Relevant & \multicolumn{2}{|c|}{ Margin } & \multicolumn{2}{|c|}{ Severity } \\
Disturbance & $N+M$ & $\mu$ & $\sigma$ & \multicolumn{2}{|c|}{$\mu \mathrm{Ma}_{i}(\mathrm{MW})$} \\
\hline Ma $(\mathrm{MW})$ & \multicolumn{2}{|c|}{$\sigma$} \\
\hline \hline No disturbance & 5000 & 756 & 325 & 0 & 0 \\
\hline Synch. Cond. tripping & 4527 & 667 & 316 & 99 & 38 \\
Line tripping & 4610 & 662 & 340 & 97 & 105 \\
Generator tripping & 4041 & 439 & 302 & 355 & 88 \\
\hline
\end{tabular}




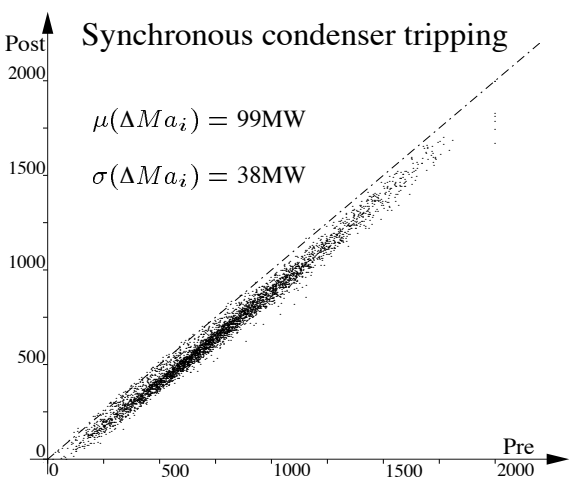

(a)

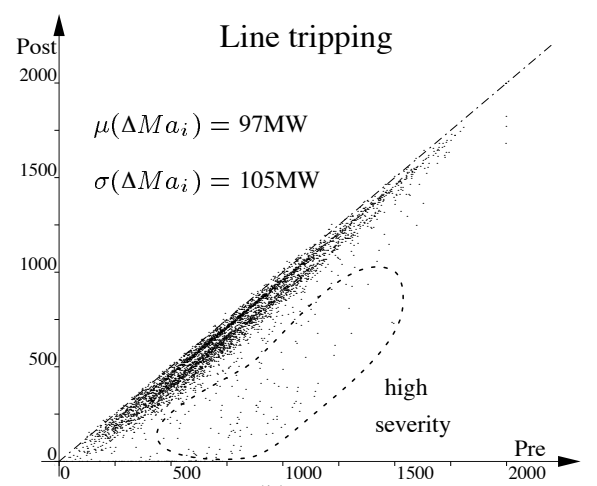

(b)

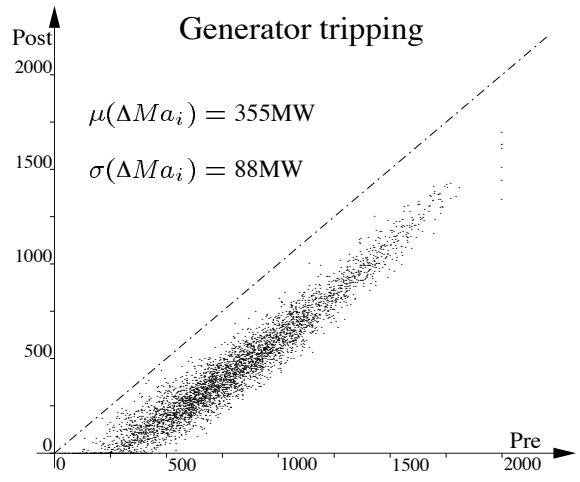

(c)

Figure 6. Scatter plots of pre-vs post-contingency margins (MW total in the study region)

mean severity is of $99 \mathrm{MW}$ and its standard deviation is only $38 \mathrm{MW}$. Considering the standard deviation of $316 \mathrm{MW}$ of the post-contingency margin for this contingency (see Table 2) we observe that it is indeed much simpler to "explain" the severity than the post-contingency margin in this case.

Actually, given the lower bound on precision of the margin computation itself $(15 \mathrm{MW})$ and taking into account the random influence of the load distribution (up to 60MW) it would not make sense to try to further refine this information; thus the regression model reduces here to its most simple constant shape : $F_{i}\left(a_{1}(o), \ldots, a_{n}(o)\right)=99 \mathrm{MW}$.

\subsubsection{LINE TRIPPING AND CORRESPONDING REGRESSION TREE}

Unfortunately, all contingencies are not so simple. For example, considering the scatter plot corresponding to the line tripping contingency (loss of Circuit 1 of the double circuit line shown in Fig. 5), we observe that its variability is much higher although its mean severity ( $97 \mathrm{MW})$ is similar to that of the preceding contingency. In particular, the operating states shown on the lower part of the scatter plot of Fig. 6b correspond to very large severities (e.g. $\Delta\left(M a_{i}\right) \geq 500 \mathrm{MW}$ ). Thus, in the present case a less trivial model is required to predict the severity.

To illustrate the regression trees, we will describe in detail their application. However, for the sake of simplicity, and noting that the lost line is an important one allowing to import power to the load region from Plant 2, we will use only two candidate attributes : the total reactive reserve in Plant 2 and the logical status (in or out) of Circuit 2.

We consider the 4610 relevant states (i.e. where Circuit 1 is in operation) and put aside 1835 test states to assess the reliability of the tree. The remaining 2775 states are used as a learning set to build a regression tree, yielding the 3 -level tree represented in Fig. 4. Each node of the tree is represented by a box containing a graphical representation of the distribution of values of $\Delta \mathrm{Ma}_{i}$ in the learning set at this node, together with its sample mean value and standard deviation, and the number of its learning states (e.g. $N=2775$ at the top-node).

The tree is built in a top-down fashion, starting at the topnode, where the reactive reserve in Plant 2 is automatically selected as the best test attribute, together with its threshold value of 191Mvar. This test is determined by the tree building method so as to provide a maximum amount of information of the contingency severity in the learning set. Once the test has been selected, the learning set is split into two subsets, corresponding respectively to 1219 and 1556 states. This reduces the variance from $106^{2}=11236$ at the top-node to a mean value of $\frac{1219}{2775} 67^{2}+\frac{1556}{2775} 116^{2}=9517$ at its successors.

Proceeding at both successors, we see that the selected test consists of checking whether Circuit 2 is in operation or not, which allows us to further reduce significantly the overall variance to a mean value of $\frac{1146}{2775} 21^{2}+\frac{73}{2775} 80^{2}+\frac{1464}{2775} 38^{2}+\frac{92}{2775} 155^{2}=$ 1817. Thus, the regression tree explains $100 \times\left(1-\frac{1817}{11236}\right)=$ $84 \%$ of the variance of the severity.

Once the tree has been constructed, it may be used to estimate the contingency severity of an unknown state : to this end, we direct the state from the top-node to the appropriate successor according to its reactive reserve and further to a terminal node according to the status of Circuit 2. There, the severity is estimated by the mean severity of the corresponding learning states.

Let us discuss the physical interpretation of the tree.

L1. The left most terminal node corresponds to 1146 precontingency states whose reactive reserve is smaller than 191Mvar while both circuits are in operation. The tree tells us that under these conditions the loss of one circuit is not severe at all, yielding a mean severity of $37 \mathrm{MW}$ with a standard deviation of $21 \mathrm{MW}$, i.e. of the same order than the margin computation error.

L4. Conversely, the right most terminal node tells us that if the reserve is rather high and already one circuit is out of operation, then the loss of the other circuit is a very severe contingency, leading to an expected reduction in load power margin of 547MW.

L3. The slightly higher mean value and standard deviation of the severity at this node as compared to node L1, translates the fact that higher reactive reserves lead also to higher severities of the loss of a single circuit out of two.

L2. This node is similar to L4, in that the only circuit in operation is lost; it tells us however that in this case the severity is not so important since not so much reactive reserve is available in Plant 2.

Although it might admittedly be further improved by further developing some of its terminal nodes on the basis of other attributes able to reflect complementary information, we will see that this tree, as simple as it is, provides a quite accurate estimate of the post-contingency load-power margin. To assess its accuracy, we apply it to estimate the contingency severity of the 1835 independent test states not used to build the tree. The difference between this estimate and the "actual" value precomputed by simulation yields an overall mean error of $-0.5 \mathrm{MW}$ and standard deviation of 43.6MW.

Of course, the same error distribution is also obtained if we subtract the estimated severity from the pre-contingency LPM so as to estimate the post-contingency LPM, according to eqn. (1). This is illustrated in Fig. 7a showing the scatter plot of the 


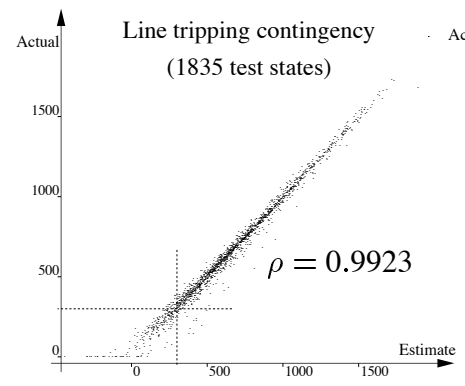

(a)

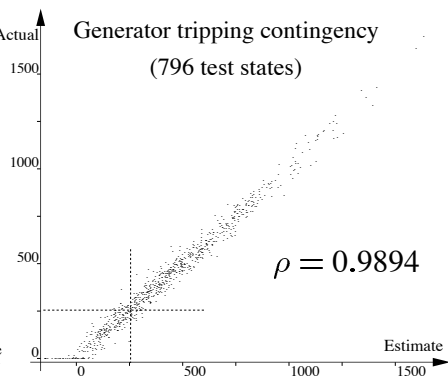

(b)
Figure 7. Computed vs estimated post-contingency LPMs

test states in terms of their "actual" post-contingency load power margin and the estimated value of the latter. We notice that the estimated and actual LPMs are highly correlated. The correlation coefficient of 0.9923 indicates that overall the estimate is able to "explain" $98.5 \%$ of the variance of the post-contingency margin. This suggests that a very simple model may indeed provide valuable information about complex quantities such as post-contingency margins. To further fix ideas, we indicate that when using the estimated margin to classify the test states with respect to a threshold of $300 \mathrm{MW}$ (considering as unsecure the states for which the post-contingency margin is smaller than this threshold) leads to a classification error rate of $2.56 \%$.

Giving a closer look at Fig. 7a, we observe that where the "actual" margin values are zero (i.e. if the contingency leads to a mid-term voltage instability without load increase), the estimated margin values become negative. Such negative margin values could for example be interpreted as the amount of emergency load shedding required to prevent a voltage instability.

To illustrate the possibility of exploiting the regression models to asses the impact of multiple contingencies, we use the tree to estimate the severity of the loss of two circuits.

We start with the hypothesis that the circuits are both in operation, and apply the tree to determine the severity of the first outage. If a new mid-term steady state is reached before the second circuit is lost, yielding in particular a change in the reactive reserve in Plant 2, we may apply the regression tree a second time in order to evaluate the impact of the loss of the second circuit. Notice that if the reactive reserve was initially smaller than 191Mvar, we might assume that it will remain smaller than this threshold after the loss of a circuit. Thus, in this particular case it is not necessary to recompute the steady state reached after the first outage to predict the effect of the second one; the severity of the double circuit outage is then estimated to $37+292=329 \mathrm{MW}$. Remarkably, this is actually very close to the true mean severity $(327 \mathrm{MW})$ of the double circuit outage, as obtained in the same conditions by direct margin computation.

\subsubsection{GENERATOR TRIPPING AND CORRESPONDING REGRES- SION TREE AND MULTILAYER PERCEPTRON}

The third contingency - illustrated in Fig. 6c - consists of the loss of a generator ( $\approx 700 \mathrm{MVA})$ in Plant 1 shown in Fig. 5 , which is located in the "center" of the load region. We observe from the scatter plot that this contingency is much severer than the two preceding ones $\left(\mu\left(\Delta M a_{i}\right)=355 \mathrm{MW}\right)$ and rather variable $\left(\sigma\left(\Delta M a_{i}\right)=88 \mathrm{MW}\right)$. Moreover, in contrast to the line tripping contingency it is difficult to suggest a priori a small number of parameters liable to "explain" this contingency.

Thus we will illustrate the overall systematic approach suggested in $\S 2$, combining regression trees and multilayer perceptrons. To validate the model $F_{i}\left(a_{1}(o), \ldots, a_{n}(o)\right)$, we keep aside 796 test states among the 4041 relevant states of this contingency, and we use the remaining 3245 states as learning set. So as to avoid missing some important information, a rather large list of 138 pre-disturbance candidate attributes is used.

The first step consists of building a regression tree. This selects among the 138 candidate attributes those which are most strongly correlated with the severity. In the present case, 15 test attributes are selected, comprising by decreasing order of importance the reactive flow through the $400 / 225 \mathrm{kV}$ transformers in substation 2, the total reactive EHV compensation of the region, the active flow through $400 / 225 \mathrm{kV}$ transformers in Plant 1 substation and the reactive reserve available in this plant. The regression tree remains however quite simple, since it is composed of 18 test nodes and 19 terminal nodes.

The model is further refined by exploiting the continuous modelling capabilities of multilayer perceptrons. To this end, we use a multilayer perceptron with 15 input neurons, corresponding to the 15 attributes selected by the tree, 20 (this number is arbitrarily fixed) hidden neurons, and a single output neuron corresponding to the severity appropriately normalized in the interval $[-0.5 \ldots 0.5]$. The BFGS procedure, applied to adjust the 340 weights of the latter model so as to reduce the overall MSE in the learning set, converged after 305 passes through the learning set. Using the multilayer perceptron to approximate the value of the severity of the test states yields a mean error of $-0.8 \mathrm{MW}$ and standard error deviation of $43 \mathrm{MW}$. Figure $7 \mathrm{~b}$ shows the scatter plot of the pre-computed post-contingency margin vs. the estimated one using eqn. (1).

Further, the use of this margin to classify test states with respect to to a threshold of $255 \mathrm{MW}$ (see Fig. $7 \mathrm{~b}$ ) yields an error rate of $4.9 \%$. Given the lower bound of the margin computation accuracy (and the thereby induced error rates shown in Table 1), we conclude that the proposed approach yields a very satisfactory level of accuracy, for all three contingencies.

\section{DISCUSSION}

We will first discuss computational feasibility and then concentrate on potential uses of the approach.

\subsection{Computational feasibility}

It is appropriate to distinguish among the three steps of the approach indicated in Fig. 1. The most bulky part concerns the data base generation and the pre-computation of the LPMs. For example, the computation of the 135,000 LPMs of our data base took all in all about 1 month CPU on a SUN Sparc 10 workstation (18MFLOPS). However, using several high-end workstations in parallel, the elapsed time may be easily reduced to less than one day. The second step, consisting of extracting the regression models from a data base, is much faster, although it remains offline task. To fix ideas, on the same hardware it would typically take some minutes to build a regression tree and some hours to optimize the weights of a multilayer perceptron. Finally, the on-line use of the trees or the perceptrons is extremely fast, and would typically take less than a millisecond per margin estimation.

\subsection{Potential uses}

In the off-line study environment (planning or operational planning) the above approach enables security experts to gain valuable insight into the physical behavior of their system, by allowing them to identify the predominant factors and conditions which determine contingency severities. This is, by itself, a very important outcome of the method. On the other hand, once the synthetic models have been extracted and validated they may be used for security analysis and control, in particular in the on-line operation context as is further discussed below. 


\subsubsection{ANALYSIS}

Security assessment is decomposed into successive steps.

1. General system robustness is assessed by the pre-contingency LPM, which we suggest to compute using one of the "system theory" approaches proposed in the literature.

2.If the latter margin is sufficiently large, we propose to analyze each contingency in terms of its impact on the LPM, using the above approximate models. The regression trees provide also sensitivity type of information about the most influencing system parameters.

3. If all post-contingency margins are large enough, we should search for combinations of contingencies most likely to lead to voltage collapses.

The last problem may be reformulated as the search for the shortest path to insecurity, where the length of the path is determined by the probability of the corresponding combination of contingencies leading from the present state to a situation where the LPM is reduced below an acceptable threshold. This latter analysis aims thus at completing "classical" security assessment, which often leads to rather uninformative answers, by a more informative identification of the system weak points. This is the adaptation to voltage security assessment of the means-ends analysis proposed by Talukdar and Christie [14].

\subsubsection{CONTROL}

Since the regression trees provide means to identify the variables which influence most strongly the severity of a contingency, they may suggest which preventive control alternatives the operator could apply to reduce the latter severity. However, at the present stage it is not clear how this could be achieved so as to actually increase security, i.e. without leading to simultaneous reduction of pre-contingency LPM for example.

On the other hand, once the most dangerous events or sequences of events have been identified, more refined simulations may be applied to pre-determine possible corrective control actions, e.g. according to the approach proposed in [7].

\section{CONCLUSION}

In this paper we have proposed a novel approach to voltage security assessment. It exploits non-parametric regression techniques to extract simple and at the same time reliable models of the severity of a contingency, in terms of the incumbent reduction in LPM.

The soundness and computational feasibility of the idea have been illustrated on a realistic model of a large voltage stability limited power system, showing very promising results and in particular the ability to furnish interesting security information. It is however too early to draw definite conclusions about the possible uses of the above regression approach in planning and operation. In particular, we quote the following, still open questions.

Which kind of attributes may provide appropriate accuracy / flexibility compromises for regression models ?

In particular, is it possible to use attributes which are independent or controllable system parameters ?

How sensitive are the regression models to the way load power margins are defined, e.g. to stability criteria, models and individual load participations?

How to devise a search strategy exploiting the approximate models to identify dangerous combinations of outages?

On the other hand, we recall that the flexibility of the computer based learning approach allows us to account for any required modelling sophistication, and to study the influence on security of any kind of system parameters and models. The regression trees provide interpretable information, which may receive various interesting uses. In this respect the proposed method is a natural complement to the decision tree based approach to voltage security assessment previously proposed [15].

\section{ACKNOWLEDGEMENTS}

This work was accomplished in collaboration with Electricite de France. I am pleased to acknowledge the valuable discussions with my colleagues Yannick Jacquemart, Jean-Noël Marquet, and Patrick Pruvot of Electricité de France.

\section{REFERENCES}

[1] IEEE System Dynamic Performance Subcommittee of the power system engineering committee of the PES. Voltage stability of power systems : concepts, analytical tools, and industry experience. Technical Report 90TH0358-2-PWR, IEEE, 1990.

[2] North American Electricity Reliability Council. Survey of the voltage collapse phenomenon - Summary of the Interconnection Task Force. Technical report, NERC, 1991.

[3] C. W. Taylor. Power System Voltage Stability. McGraw-Hill, 1993.

[4] C. Lemaître, J. P. Paul, J. M. Tesseron, Y. Harmand, and Y. S. Zhao. An indicator of the risk of voltage profile instability for realtime control applications. IEEE Trans. on Power Syst. PWRS-5, no. 1, pp. 148-0.861, Feb. 1990.

[5] T. Van Cutsem. A method to compute reactive power margins with respect to voltage collapse. IEEE Trans. on Power Syst. PWRS-6, no. 2,pp. 145-0.856, Feb. 1991.

[6] F. L. Alvarado, Y. Hu, C. Rinzing, and R. Adapa. Vizualization of spatially differentiated security margins. In Proc. of the 11th Power Systems Computation Conf., pp. 519-525, Aug-Sept 1993.

[7] T. Van Cutsem, Y. Jacquemart, J.N. Marquet, and P. Pruvot. A comprehensive analysis of mid-term voltage stability. Paper \# SM94056 to be presented at the IEEE PES Summer Meeting 1994. To appear in IEEE Trans. on Power Syst., 1995.

[8] L. Wehenkel and M. Pavella. Decision tree approach to power system security assessment. Int.J. of Elec. Power and Energy Syst. 15, no. 1, pp. 13-36, 1993.

[9] L. Wehenkel, T. Van Cutsem, M. Pavella, B. Heilbronn, and P. Pruvot. Machine learning, neural networks and statistical pattern recognition for voltage security : a comparative study. In Proc. of ISAP'94, 1994.

[10] L. Breiman, J. H. Friedman, R. A. Olshen, and C. J. Stone. Classification and Regression Trees. Wadsworth Int., 1984.

[11] J. M. Zurada. Introduction to artificial neural systems. West Publishing, 1990.

[12] J. Hertz, A. Krogh, and R. G. Palmer. Introduction to the theory of neural computation. Addison Wesley, 1991.

[13] Y. Harmand, M. Trotignon, J. F. Lesigne, J. M. Tesseron, C. Lemaître, and F. Bourgin. Analyse d'un cas d'écroulement en tension et proposition d'une philosophie de parades fondées sur des horizons temporels différents. In CIGRE Report 38/39-02, Paris, Aug. 1990.

[14] R. D. Christie and S. N. Talukdar. Discrete approximations and means-ends analysis for static security assessment. In Proc. of the 2nd Symposium on expert systems application to power systems, pp. 177-0.882, July 1989.

[15] L. Wehenkel, T. Van Cutsem, M. Gilliard, M. Pavella, B. Heilbronn, and M. Goubin. Decision trees for preventive voltage stability assessment. In Proc. of the 2nd Int. NSF Workshop on Bulk Power System Voltage Phenomena - Voltage Stability and Security, Deep Creek Lake, Ma, pp. 217-228, Aug. 1991.

Louis Wehenkel was born in Nürnberg, Germany, in 1961. He received the Electrical (Electronics) engineering degree in 1986 and the $\mathrm{Ph} . \mathrm{D}$. degree in 1990 both from the University of Liège, Belgium, where he is a senior research assistant of the Fonds National de la Recherche Scientifique. His research interests lie mainly in the application of artificial intelligence methodologies to power system security assessment. 